\title{
Optical Imaging with a Cathepsin B Activated Probe for the Enhanced De- tection of Esophageal Adenocarcinoma by Dual Channel Fluorescent Upper GI Endoscopy
}

\author{
Peiman Habibollahi' ${ }^{1}$, Jose-Luiz Figueiredo², Pedram Heidari', Austin M Dulak³, Yu Imamura4, Adam J. \\ Bass $^{3}$, Shuji Ogino3, ${ }^{3}$, Andrew T Chan 5 and Umar Mahmood ${ }^{1 凶}$ \\ 1. Division of Nuclear Medicine and Molecular Imaging, Department of Radiology, Massachusetts General Hospital, \\ 2. Division of Cardiovascular Medicine, Department of Medicine, Brigham and Women's Hospital, \\ 3. Department of Medical Oncology, Dana-Farber Cancer Institute, \\ 4. Department of Pathology, Brigham and Women's Hospital, \\ 5. Gastrointestinal Unit, Massachusetts General Hospital, Harvard Medical School, Boston, MA
}

Corresponding author: Umar Mahmood, MD. PhD., Division of Nuclear Medicine and Molecular Imaging, Department of Radiology, Massachusetts General Hospital, Harvard Medical School, Boston, MA. Email: umahmood@mgh.harvard.edu Phone: 617.726.6477; Fax: 617.726.6165.

(C) Ivyspring International Publisher. This is an open-access article distributed under the terms of the Creative Commons License (http://creativecommons.org/ licenses/by-nc-nd/3.0/). Reproduction is permitted for personal, noncommercial use, provided that the article is in whole, unmodified, and properly cited.

Received: 2012.01.13; Accepted: 2012.01.24; Published: 2012.02.16

\begin{abstract}
Despite significant advances in diagnosis and treatment, the prognosis of esophageal adenocarcinoma remains poor highlighting the importance of early detection. Although white light (WL) upper endoscopy can be used for screening of the esophagus, it has limited sensitivity for early stage disease. Thus, development of new imaging technology to improve the diagnostic capabilities of upper $\mathrm{Gl}$ endoscopy for early detection of esophageal adenocarcinoma is an important unmet need. The goal of this study was to develop a method for the detection of malignant lesions in the esophagus using WL upper endoscopy combined with near infrared (NIR) imaging with a protease activatable probe (Prosense750) selective for cathepsin B (CTSB). An orthotopic murine model for distal esophageal adenocarcinoma was generated through the implantation of OE-33 and OE- 19 human esophageal adenocarcinoma lines in immunocompromised mice. The mice were imaged simultaneously for WL and NIR signal using a custom-built dual channel upper Gl endoscope. The presence of tumor was confirmed by histology and target to background ratios (TBR) were compared for both WL and NIR imaging. NIR imaging with ProSense750 significantly improved upon the TBRs of esophageal tumor foci, with a TBR of $3.64 \pm 0.14$ and $4.50 \pm 0.11$ for the OE-33 and OE-19 tumors respectively, compared to $0.88 \pm 0.04$ and $0.8 I \pm 0.02$ TBR for WL imaging. The combination of protease probes with novel imaging devices has the potential to improve esophageal tumor detection by fluorescently highlighting neoplastic regions.
\end{abstract}

Key words: Esophageal Adenocarcinoma; Cathepsin B; Prosense750; Near Infrared Imaging.

\section{Introduction}

The recent epidemiological shift of esophageal cancer in western countries from squamous cell carcinoma towards adenocarcinoma, which arises from prolonged Barrett's esophagus, is reflected in the in- creased incidence of distal esophageal adenocarcinoma [1]. In recent decades esophageal adenocarcinoma has had the fastest growing incidence rate compared to any other cancer [2]; during the last 40 
years its incidence has increased 300\% in the United States [3]. Despite current advances in diagnosis and treatment as well as our understanding of underlying mechanisms leading to Barrett's esophagus and adenocarcinoma, the prognosis of the patients remain poor with a 5 -year survival of about $15 \%[4,5]$. Like many other malignancies, early detection can improve the prognosis in these patients. However, current modalities for the detection of high grade dysplasia or early stage cancer is challenging as the appearance of the tissue at these stages of the disease can be difficult to differentiate from normal mucosa using conventional white light (WL) endoscopy. Moreover, random biopsies to identify dysplastic regions within macroscopically normal appearing esophagus can miss early cancers due to the patchy nature of the dysplasia [6].

Near Infrared (NIR) optical imaging combined with fluorescent probes has been applied towards more sensitive detection of a range of cancers including colon, endometrial, breast, lung and prostate cancer [7-13]. A class of optical imaging agents that are "smart", NIR protease activatable agents become brightly fluorescent in areas of increased cathepsin B (CTSB) expression [8, 14]. The agents are optically silent in their native state and markedly increase their fluorescent intensity after enzyme-mediated release of fluorochromes, resulting in signal amplification at the site of release. Therefore, they offer a high tumor to background ratio compared with nonspecific agents, due to their selective activation.

CTSB is a cysteine protease that actively participates in the proteolytic degradation of the extracellular matrix. It is believed that CTSB plays a critical role in tumor progression, and shows an overexpression in certain tumors of the lung, breast, stomach, colon, esophagus and prostate [15-17] as well as esophageal adenocarcinoma [18]. In the current study we have developed an orthotopic murine model of distal esophageal adenocarcinoma. We hypothesized that NIR endoscopy with protease activatable agents can be used as an adjunct to WL endoscopy for sensitive detection of tumor foci in our esophageal adenocarcinoma model. We demonstrated the ability of Prosense750 in delineating esophageal tumor foci with a high tumor to background ratio (TBR) using our in-house custom made dual-channel (WL and NIR) upper GI endoscope system.

\section{Methods}

\section{NIR activatable probe}

The imaging probe used for this study is a commercially available protease-activatable NIR flu- orescent probe, Prosense750 (Perkin-Elmer, Waltham, MA). The probe's structure consists of a synthetic graft polymer composed of poly-L-lysine that is sterically protected by multiple methoxypolyethylene glycol side chains. Conjugated onto this lysine backbone are multiple NIR fluorochromes whose fluorescence is quenched by their proximity to one another in a phenomenon similar to fluorescence resonance energy transfer. Following a cleavage event by proteases that degrade the lysine backbone, the fluorochromes are released and thus regain their fluorescent properties, increasing the NIR signal intensity.

\section{Cell culture}

Human esophageal adenocarcinoma cancer cell lines OE-33 and OE-19 (both from Sigma-Aldrich) were grown in RPMI, containing L-glutamine (CellGro by Mediatech Inc., Manassas, VA). The media were supplemented with $10 \%$ fetal bovine serum (Atlanta biologicals, Lawrenceville, GA) and 1\% Penicillin Streptomycin (CellGro by Mediatech Inc.). Cells were maintained in a humidified incubator at $37^{\circ} \mathrm{C}$ and $5 \% \mathrm{CO}_{2}$.

\section{Surgical procedure and tumor implantation}

OE-33 and OE-19 cells were grown in cell culture medium until they were $\sim 90 \%$ confluent. Cells were trypsinized, washed three times with PBS and collected by centrifugation (5min, 1200rpm, RT). Cells were diluted $25 \times 10^{6} / \mathrm{mL}$ in PBS and kept on ice until injection.

Six to 8-week old female athymic Balb/C nude mice ( 3 groups, $n=5$ each) were obtained from Charles River Laboratories International Inc., (Wilmington, MA). Mice were anesthetized by IP administration of ketamine $(100 \mathrm{mg} / \mathrm{kg})$ and xylazine $(10 \mathrm{mg} / \mathrm{kg})$. The surgical procedure was performed under a stereomicroscope (Nikon, Tokyo, Japan) with a 10:1 zoom ratio. After induction of the anesthesia, the anterior abdomen wall was swabbed with povidone-iodine solution and a midline incision below the costal margin was made to access the abdominal esophagus. Clean sterilized cotton tips were carefully used to expose the distal esophagus. Pre-cell injection average esophageal wall thickness including the mucosa and submucosa in mouse was measured to be between 400 and 500 microns. Approximately, $5 \times 10^{5}$ cells (group $\mathrm{A}=\mathrm{OE}-33$, group $\mathrm{B}=\mathrm{OE}-19$, and group $\mathrm{C}=\mathrm{PBS}$ ) were injected in $20 \mathrm{uL}$ of PBS using a 32-gauge needle into the distal esophagus proximal to the gastroesophageal junction (Figure 1). Observation of a small bulge in the esophagus wall was considered as the sign of successful implantation. 

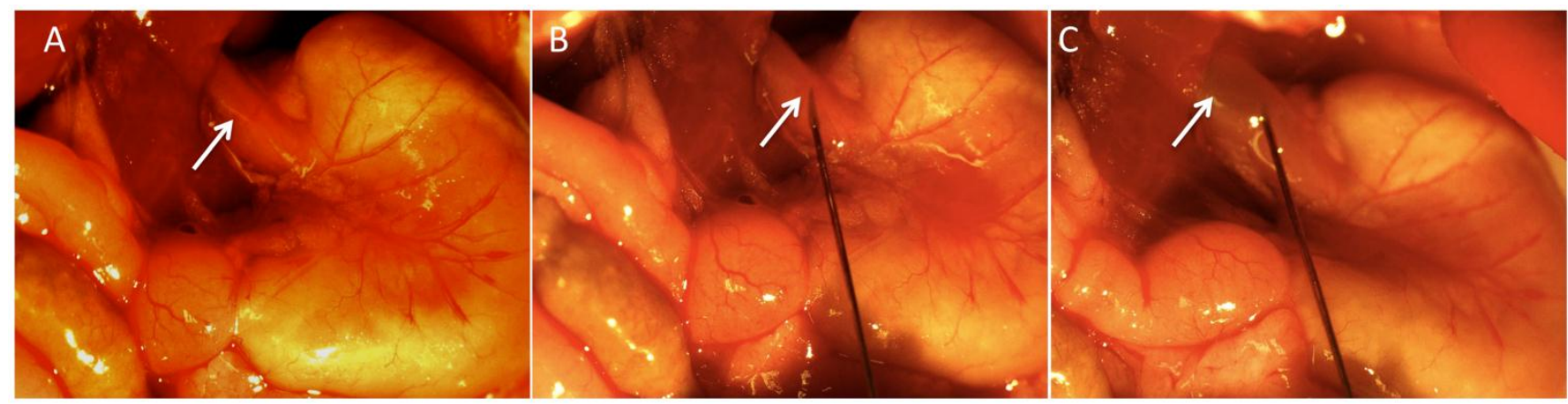

Figure I: Images acquired during the surgery to generate the orthotopic model of distal esophageal adenocarcinoma. Distal esophagus (panel A, white arrow) was exposed following a midline incision in the upper abdomen. The 32-guage needle was inserted into the esophagus wall (panel B, white arrow) and after injection, induction of a small bulge (panel $\mathrm{C}$, white arrow) was considered as a sign of successful injection.

After careful observation of the animals over several minutes to ensure that the cells did not leak from the injection site, the abdominal wall and skin were closed using 5-0 prolene sutures. The animals were observed until recovered from anesthesia and were kept for 4 weeks under standard laboratory conditions with free access to water and food in a 12-hour light/dark schedule until tumors reached a size of 2-3 $\mathrm{mm}$ in diameter. Pain reduction precautions were administered according to the institutional guidelines.

\section{Small Animal Upper GI endoscopy and ex vivo imaging}

The animals received 2nmol of Prosense 750 (Perkin-Elmer, Waltam, MA) via tail vein under 2\% isoflurane anesthesia, 24 hours prior to the upper GI endoscopy. For the endoscopy, anesthesia was induced using $5 \%$ isoflurane and $100 \%$ oxygen mixture and the animals were fixed on a regulated heating plate to maintain the body temperature at $37^{\circ} \mathrm{C}$. The animals were then intubated using a 22-guage plastic catheter that was connected to a small animal respirator (Harvard apparatus, Holliston, MA) and the anesthesia was maintained by $1 \%$ isoflurane.

The upper endoscopy was performed using our in-house custom-made multichannel endoscopy system (two channels for WL and NIR); the design of the endoscope system has previously described by our group [10]. The flexible imaging catheter was gently introduced through the oropharynx while air was insufflated through the endoscope working channel by a $10 \mathrm{ml}$ syringe. Simultaneous WL and NIR images from esophagus were acquired with slow advancement of the fiberscope.

After endoscopy, the animals were immediately sacrificed using a $\mathrm{CO}_{2}$ chamber, the thorax and abdomen were opened and esophagi were dissected.
Epifluorescence imaging was performed using an in vivo multispectral whole animal optical imaging system (Carestream, Rochester, NY). A 750nm excitation filter and an $810 \mathrm{~nm}$ emission filter were used (excitation and emission ranges for the Prosense750 probe) to image the signal generated from the activated NIR probe. To evaluate the fluorescent biodistribution of the probe, non-tumor bearing mice were also dissected and the organs were imaged on the same system to find the baseline signal in the different organs including the esophagus and stomach, 24 hours after Prosense750 injection.

Quantitative measurements were performed on the acquired near-infrared images. The mean signal intensity and standard error of mean of esophagus tumor and an adjacent esophagus normal mucosa, as well as a region outside the specimen (noise), were measured on 12-bit gray-scale images using proprietary software [19]. The target (esophagus tumor) to background (normal esophagus) contrast (TBC) was calculated as follows: TBR $\%=($ ET - Noise $) /($ NE Noise) Where: $\mathrm{ET}=$ esophagus tumor, $\mathrm{NE}=$ normal esophagus. The mean signal intensity in regions-of-interest (ROI) of constant size comprising 10 $x 10$ units of width and height was measured. The tumor bearing ROI were placed with the center over the respective brightest fluorescence in the tumors; the normal esophagus ROI (background) were placed over normal esophagus tissue at a distance of three region-of-interest diameters from the tumor center; the noise was measured on the image outside the specimen. For consistency, the same investigator placed all regions-of-interest. The data is shown as mean \pm SEM. Signal intensity (SI) of esophagus tumor from animals injected with OE-33 and OE-19 cells were compared with signal intensity of esophagus injection region from animals injected with PBS in the distal esophagus, using a two-tailed Student $t$ test for 
independent samples. A p value $<0.05$ was considered to be significant.

\section{Histological confirmation and Immunohisto- chemistry}

The extracted esophagus samples were immediately transferred into formalin for fixation and further processing. The paraffin embedded blocks were prepared and slices were cut and stained. Hematoxylin and eosin (H\&E) staining was used for the confirmation of tumor presence. Consecutive slices were used for immunohistochemistry using a CTSB primary antibody. Briefly, for CTSB staining, antigen retrieval was performed, and deparaffinized tissue sections in antigen retrieval Citra Solution (Biogenex Laboratories, San Ramon, CA) were treated with microwaves in a pressure cooker (5 $\mathrm{min})$. Tissue sections were incubated with 5\% normal rabbit serum (Vector Laboratories, Burlingame, CA) in PBS (30 min). Primary antibody against CTSB [Goat polyclonal anti-CTSB (S-12), 1:1250 dilution; Santa Cruz Biotechnology, Inc., Santa Cruz, CA] was applied, and the slides were maintained at room temperature for $15 \mathrm{~min}$, followed by goat secondary antibody (Vector Laboratories) (30 min), an avidin-biotin complex conjugate (Vector Laboratories) (30 min), diaminobenzidine (5 min) and
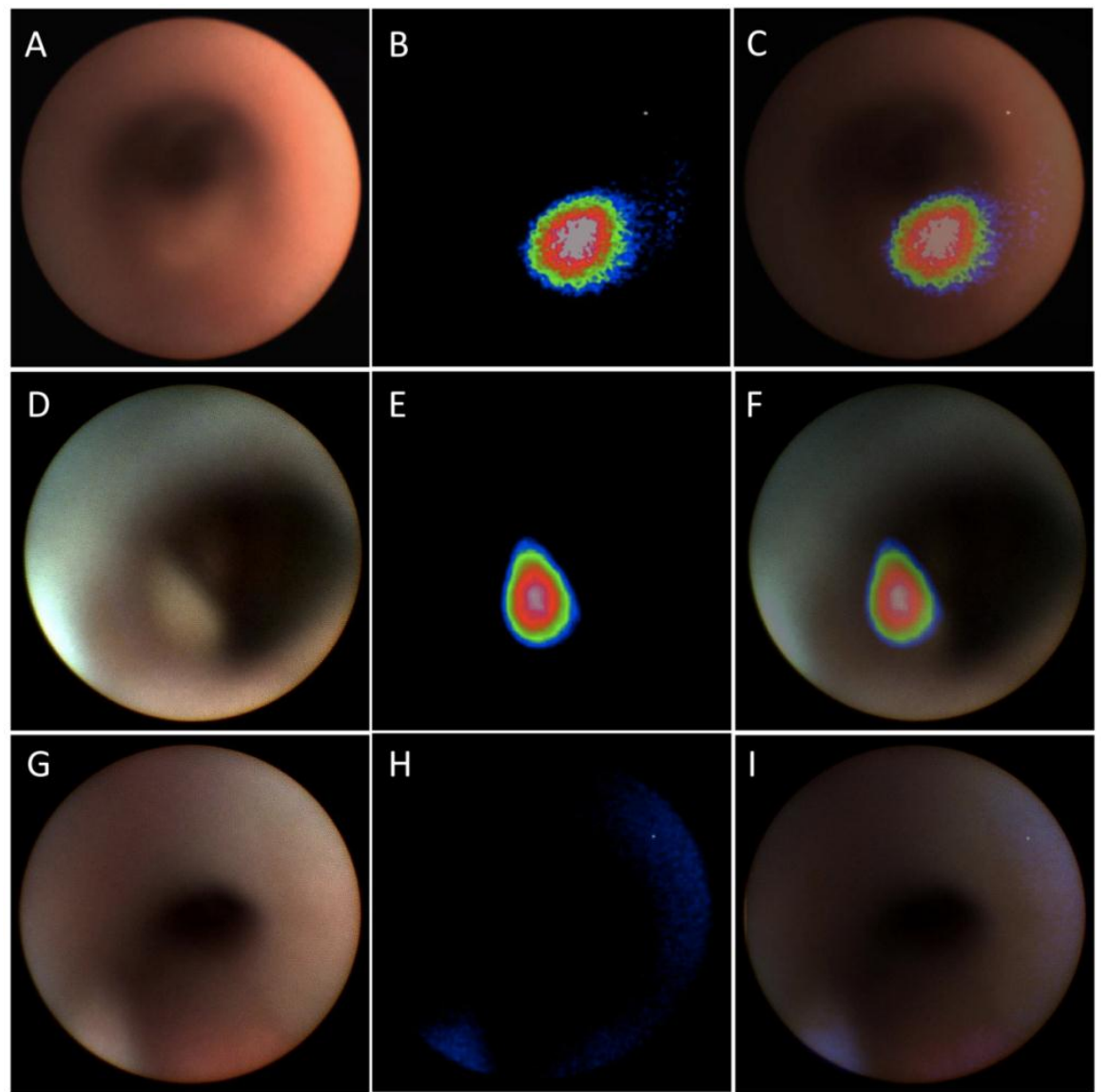

counterstained with hematoxylin. After preparation, all slides were evaluated by a pathologist for confirmation.

\section{Results}

\section{Surgical outcome and animal model}

The depth of needle penetration for cell implantation was controlled under streomicroscope visualization in order to produce sub mucosa small distal esophagus tumor nodules that mimicked early human esophagus adenocarcinoma. There were no complications or surgically related mortalities in any of the 15 mice.

\section{Small Animal Upper GI endoscopy and ex vivo epifluorescence imaging}

Fiber-optic esophagoscopy was performed in all three groups of the study, 24 hours after Prosense750 probe injection. No major complication, including perforation, was observed secondary to the endoscopy procedure. The WL images provided anatomic localization and visual clues for real time imaging while the NIR images reported upon the protease activity.

Figure 2: WL, NIR and overlay images of catheter-based upper $\mathrm{Gl}$ endoscopy from OE-33 (panel A, B and C), OE-19 (panel D, E and $F$ ) and control (panel G, $\mathrm{H}$ and $\mathrm{I}$ ) groups. Upper $\mathrm{Gl}$ endoscopy demonstrates significant elevated NIR signal at the tumor foci while in the control animals the signal is absent in this region.

The tumors were early in growth and there was no major obstruction of the lumen at the time point evaluated. All of the tumors were visible on the NIR channel in group $\mathrm{A}$ and $\mathrm{B}$ mice (OE-33 and OE-19 tumor bearing mice), while no NIR signal was detectable above background in the PBS-injected group. Due to small size of the lesions in some cases, the WL channel could not provide adequate visual contrast for detection of the tumors while they were brightly visible on the NIR channel. Figure 2 shows a sample of the WL and NIR channels and the overlay images acquired from each group.

Analysis of the NIR and WL signal and the TBR demonstrated a 
several fold increase in the NIR signal in the OE-33 and OE-19 group in comparison to the controls. The NIR TBR in the OE-33 and OE-19 were 3.64 \pm 0.14 and $4.50 \pm 0.11$, respectively, which were significantly higher compared to the control group non-elevated NIR TBR of $1.08 \pm 0.08$ ( $p<0.0001)$. The WL TBR $(0.88 \pm 0.04$ and $0.81 \pm 0.02$ for $\mathrm{OE}-33$ and OE-19 groups, respectively) was comparable to the WL TBR of $0.97 \pm 0.05$ for the control group. Figure 3 shows results of this analysis for each group separately.

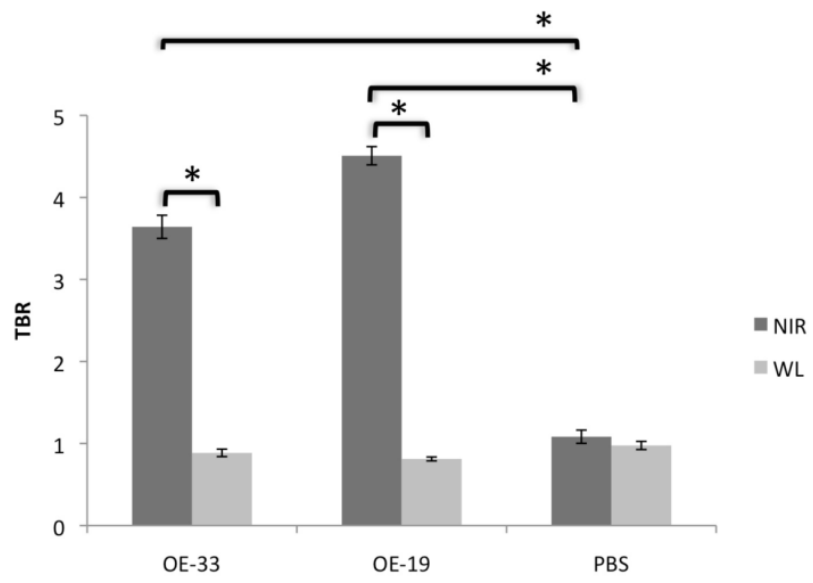

*Statistically significant difference

Figure 3: TBR of NIR optical imaging using CTSB activatable probe compared to $W L$ in different groups of the study. NIR imaging after probe administration improves the TBR in the tumor bearing mice up to 4 fold; The TBR remains close to I for both tumor and control groups.

\section{Ex vivo imaging}

Epifluorescence images demonstrated a bright fluorescent signal from the tumor site of the animals injected with the Prosense750 probe in contrast to adjacent mucosa. Figure 4 shows a sample of these images. Unlike samples from OE-33 and OE-19 groups, the esophagus samples from the control group did not show high focal fluorescence signal. The biodistribution study demonstrated high NIR signal in skin, liver and kidneys, 24 hours after probe injection but not in other organs such as esophagus, intestines or blood. Figure 5 shows a sample of our biodistribution results.

\section{Pathology}

Tumors developed at the site of direct implantation in 100\% (10/10) of animals, and formed esophagus nodules 4 weeks after tumor cell implantation. The presence of the submocusal tumor was confirmed in all samples via pathology and H\&E staining. Immunohistochemistry confirmed the elevated expression of CTSB in the tumor site but not in the controls or unaffected tissue. Figure 6 shows a sample of H\&E and immunohistochemistry for both OE-33 and control group.
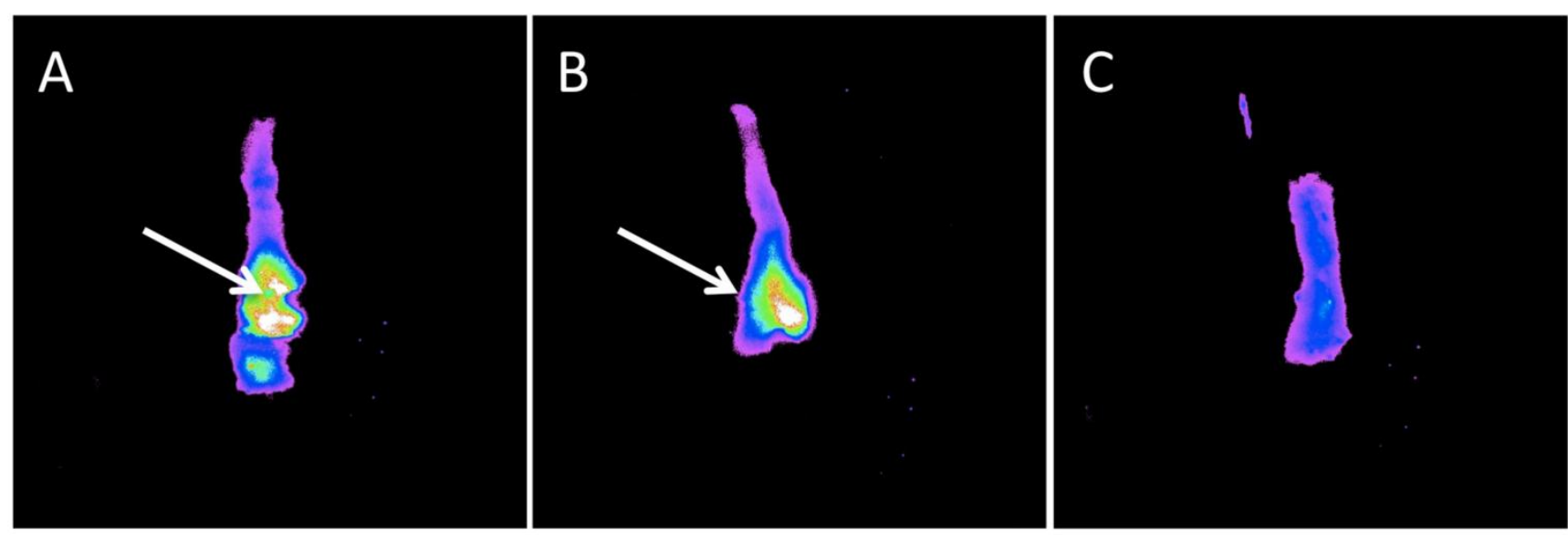

Figure 4: Ex vivo imaging of the mouse esophagus after dual channel upper GI endoscopy. Panel A and B demonstrates high focal NIR probe activity in small nodules in the esophagus of OE-33 and OE-19 tumor bearing mice, respectively. In panel C, a control mouse esophagus is displayed with no detectable focal fluorescence signal. 

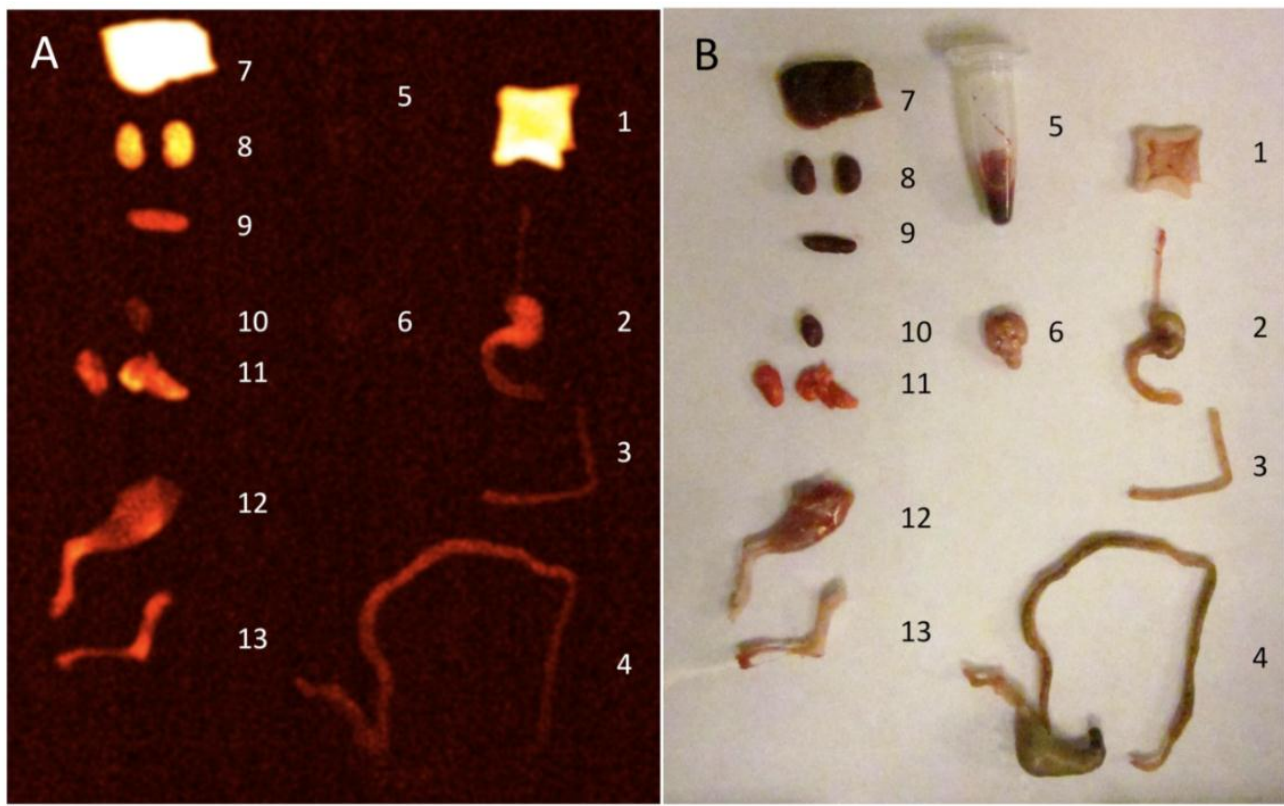

1. Skin

2. Esophagus, stomach, duodenum

3. Jejunum

4 Colon

5. Blood

6. Brain

7. Liver

8. Kidneys

9. Spleen

10. Heart

11. Lungs

12. Muscle

13. Bone

Figure 5: Imaging the fluorescent bio-distribution of the probe 24 hours after injection demonstrates high signal in skin, liver and kidneys but not in the alimentary tract.
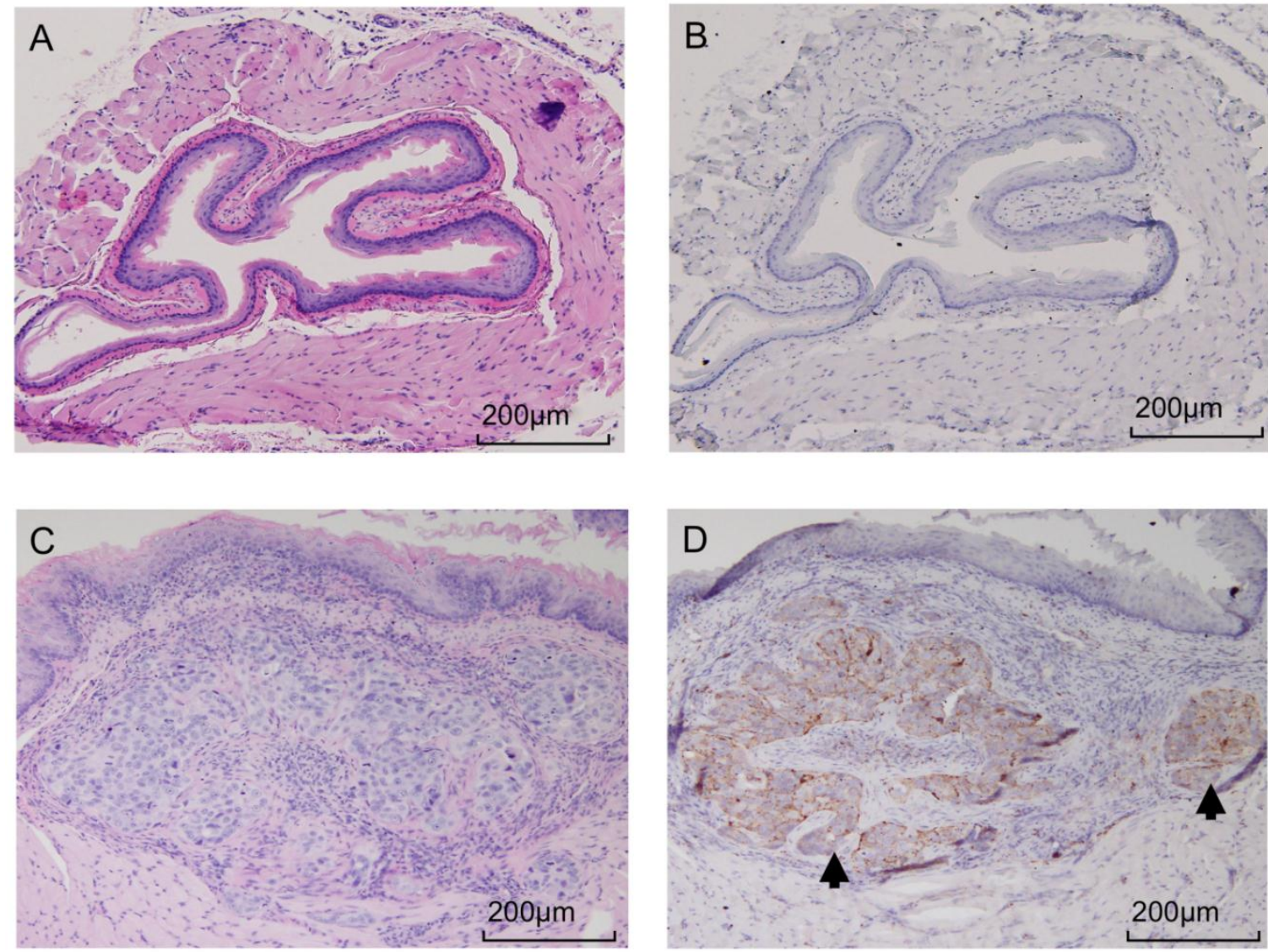

Figure 6: After upper Gl endoscopy and ex vivo imaging, presence of tumor was confirmed by pathology. Panel A shows an H\&E image from an control mouse receiving PBS injection and panel $C$ image is acquired from a tumor bearing mouse with OE- 33 cells. Consecutive slides were used for IHC, which confirmed high expression of CTSB in the tumors (panel D) but not the normal esophagus (panel B). The tumor is shown by black arrow. 


\section{Discussion}

Activatable molecularly targeted fluorescent probes combined with minimally invasive imaging systems for optical evaluation have numerous advantages over other methods for finding early disease. In particular, protease imaging selective for CTSB offers the improved detection of cancer and premalignant lesions by targeting an enzyme class widely present across most cancers and in particular adenocarcinoma of esophagus and Barrett's esophagus $[18,20]$. Such imaging yields high target to background ratios that markedly improve detection and that can help localize pathology. Near infrared imaging allows simultaneous full color spectrum WL imaging along with molecular reporting in the NIR, thereby providing anatomic landmarks and allowing the utilization of traditional anatomic cues for disease detection in addition to the fluorescent molecular reporters.

Surface weighted optical techniques such as endoscopy result in sufficient tissue NIR photon penetration to report on molecular targets up to $1 \mathrm{~cm}$ from the imaging surface. However, the pathology endoscopic systems are typically designed to detect is at the mucosal surface or within several $\mathrm{mm}$ of the surface, so this degree of light penetration is within the requirements of such an approach. The imaging systems are readily combined with traditional WL optical devices, similar to the approach demonstrated in this study, allowing rapid implementation to patient care paradigms. Many of these systems have incorporated minimally invasive resection capabilities, providing an option for therapeutic intervention immediately following NIR lesion detection. Finally, the lack of ionizing radiation increases the potential utilization for disease screening.

In this study, we performed a comparison between conventional WL upper endoscopy and NIR imaging with a protease sensitive imaging probe for esophageal adenocarcinoma foci identification, confirmed with histological validation. To our knowledge, this is the first example of dual channel murine upper GI endoscopy. We also demonstrated for the first time a novel surgical approach to implant esophageal tumors orthotopically. Our results for two human esophageal adenocarcinomas showed an approximate 3.5--4.5-fold tumor to background ratio, demonstrating a beacon effect that could be used to highlight the location of small neoplastic foci in the esophagus. Low uptake and lack of activation of the CTSB selective probe in the adjacent normal esophagus resulted in a relatively uniform low background, further improved lesion conspicuity. While formal human toxicology studies are needed for human translation, we have not seen any preclinical toxicity using the imaging probe Prosense 750 at the reported doses. Imaging using a near infrared protease activatable probe for esophageal adenocarcinoma in mice demonstrates a potential translatable path forward to improve endoscopic detection of early esophageal cancers in patients in the future.

\section{Funding Support}

This research was supported in part by National Health Institute grants U01CA143056, U01CA084301 and P50CA127003.

\section{Conflict of Interest}

The authors have declared that no conflict of interest exists.

\section{References}

1. Incarbone R, Bonavina L, Saino G, Bona D, Peracchia A. Outcome of esophageal adenocarcinoma detected during endoscopic biopsy surveillance for Barrett's esophagus. Surg Endosc. 2002; 16: 263-6.

2. Pohl H, Welch HG. The role of overdiagnosis and reclassification in the marked increase of esophageal adenocarcinoma incidence. J Natl Cancer Inst. 2005; 97: 142-6.

3. Devesa SS, Blot WJ, Fraumeni JF, Jr. Changing patterns in the incidence of esophageal and gastric carcinoma in the United States. Cancer. 1998; 83: 2049-53.

4. Provenzale D, Schmitt C, Wong JB. Barrett's esophagus: a new look at surveillance based on emerging estimates of cancer risk. Am J Gastroenterol. 1999; 94: 2043-53.

5. Yentz S, Wang TD. Molecular imaging for guiding oncologic prognosis and therapy in esophageal adenocarcinoma. Hosp Pract (Minneap). 2011; 39: 97-106.

6. Parsons S. Are we missing Gastro-Oesophageal Cancer at Endoscopy? Ann R Coll Surg Engl. 2010; [Epub ahead of print].

7. Sheth RA, Upadhyay R, Stangenberg L, Sheth R, Weissleder R, Mahmood U. Improved detection of ovarian cancer metastases by intraoperative quantitative fluorescence protease imaging in a pre-clinical model. Gynecol Oncol. 2009; 112: 616-22.

8. Weissleder R, Tung $\mathrm{CH}$, Mahmood U, Bogdanov A, Jr. In vivo imaging of tumors with protease-activated near-infrared fluorescent probes. Nat Biotechnol. 1999; 17: 375-8.

9. Figueiredo JL, Alencar H, Weissleder R, Mahmood U. Near infrared thoracoscopy of tumoral protease activity for improved detection of peripheral lung cancer. Int J Cancer. 2006; 118: 2672-7.

10. Funovics MA, Alencar H, Su HS, Khazaie K, Weissleder R, Mahmood U. Miniaturized multichannel near infrared endoscope for mouse imaging. Mol Imaging. 2003; 2: 350-7.

11. Funovics MA, Weissleder R, Mahmood U. Catheter-based in vivo imaging of enzyme activity and gene expression: feasibility study in mice. Radiology. 2004; 231: 659-66.

12. Hama Y, Urano Y, Koyama Y, Choyke PL, Kobayashi H. Activatable fluorescent molecular imaging of peritoneal metastases following pretargeting with a biotinylated monoclonal antibody. Cancer Res. 2007; 67: 3809-17.

13. Hama Y, Urano Y, Koyama Y, Gunn AJ, Choyke PL, Kobayashi $H$. A self-quenched galactosamine-serum albumin-rhodamineX conjugate: a "smart" fluorescent molecular imaging probe synthesized with clinically applicable material for detecting 
peritoneal ovarian cancer metastases. Clin Cancer Res. 2007; 13: 6335-43.

14. Mahmood U, Wallace MB. Molecular imaging in gastrointestinal disease. Gastroenterology. 2007; 132: 11-4.

15. Sloane BF. Cathepsin B and cystatins: evidence for a role in cancer progression. Semin Cancer Biol. 1990; 1: 137-52.

16. Yang ZQ, Imoto I, Fukuda Y, Pimkhaokham A, Shimada Y, Imamura $\mathrm{M}$, et al. Identification of a novel gene, GASC1, within an amplicon at 9p23-24 frequently detected in esophageal cancer cell lines. Cancer Res. 2000; 60: 4735-9.

17. Krueger S, Kalinski T, Wolf H, Kellner U, Roessner A. Interactions between human colon carcinoma cells, fibroblasts and monocytic cells in coculture--regulation of cathepsin B expression and invasiveness. Cancer Lett. 2005; 223: 313-22.

18. Hughes SJ, Glover TW, Zhu XX, Kuick R, Thoraval D, Orringer $\mathrm{MB}$, et al. A novel amplicon at 8p22-23 results in overexpression of cathepsin B in esophageal adenocarcinoma. Proc Natl Acad Sci U S A. 1998; 95: 12410-5.

19. Upadhyay $R$, Sheth RA, Weissleder R, Mahmood U. Quantitative real-time catheter-based fluorescence molecular imaging in mice. Radiology. 2007; 245: 523-31.

20. Lin L, Aggarwal S, Glover TW, Orringer MB, Hanash S, Beer DG. A minimal critical region of the 8 p22-23 amplicon in esophageal adenocarcinomas defined using sequence tagged site-amplification mapping and quantitative polymerase chain reaction includes the GATA-4 gene. Cancer Res. 2000; 60: 1341-7. 